\title{
Suggestive Evidence of Slc2a9 Polymorphisms Association in Gouty Malay Males
}

\author{
Mahfudzah $A^{\mathrm{a}}$, Nazihah MYa, Wan Syamimee WG ${ }^{\mathrm{b}}$, Huay Lin $\mathrm{T}^{\mathrm{a}}$, Wan Rohani WT \\ aHuman Genome Center, School of Medical Sciences, Universiti Sains Malaysia, Kelantan, Malaysia \\ bDepartment of Medicine, School of Medical Sciences, Universiti Sains Malaysia, Kelantan, Malaysia \\ 'School of Diagnostic and Biomedical Sciences, Faculty of Medicine and Health Sciences, Universiti Sultan \\ Zainal Abidin, Terengganu, Malaysia.
}

\section{ABSTRACT}

Introduction: Solute carrier family 2, member 9 (SLC2A9) is thought to be an important urate transporter that influences the excretion and reabsorption of serum uric acid, thus has a strong effect on serum urate and risk of gout. SLC2A9 polymorphisms have been extensively studied in various populations in association with gout development. Our aim was to test for association of SLC2A9 SNPs with gout in Malay males. Methods: 78 gouty patients and 82 normal subjects were recruited and genotyped for $r s 3733591, r s 5028843$ and $r s 11942223$ using PCR-RFLP technique. Single association and haplotype association analyses were conducted using SHEsis online software. Results: rs3733591 and rs5028843 showed association with gout with p value of 0.020 and 0.036 , respectively, whilst rs11942223 yielded no association with $p$ value of 0.08 with trend towards susceptibility projecting by $O R=3.547,3.667$ and 2.732 , respectively. It is noteworthy that haplotype $1 / 1 / 1$ conferred protection in gout with $p$ value $0.004(\mathrm{OR}=0.324$ [0.147-0.716]). Conclusion: This study might suggest an evidence of association of SLC2A9 SNPs with gout among Malay males.

KEYWORDS: Gout, SLC2A9, Malay males

\section{INTRODUCTION}

Gout is a clinical syndrome characterised by a distinctive pattern of acute arthritis associated with a disorder of urate kinetics manifesting as hyperuricaemia. The acute arthritis is caused by tissue inflammatory reaction to the formation of monosodium urate crystals that mainly affects man. ${ }^{1}$ The incidence and prevalence of gout or hyperuricaemia are rising significantly worldwide over the past 20 years ${ }^{2,3}$ including Malaysia.

In New Zealand, gout affects $9.3-13.9 \%$ of Maori men and $14.9 \%$ of Pacific Islanders men; 4,5 yielding the highest rate worldwide. Maori and Pacific Island populations also have high rates of severe gout, with frequent tophaceous disease and accelerated joint damage. ${ }^{5}$ Prevalence of gout among Caucasian men in Westernized countries is only around $1-4 \%{ }^{6}$ Study by Chang et al. in 2001 showed the prevalence of gout is also estimated to be $4.4 \%$ in Hans Chinese men. ${ }^{7}$ In 2011, a hospital-based study has shown the most likely

Corresponding author:

Dr Wan Rohani Wan Taib

School of Diagnostic and Biomedical Sciences,

Faculty of Health Sciences

Universiti Sultan Zainal Abidin Kampus Gong Badak

Kuala Terengganu 21300

Terengganu, Malaysia

Tel: (h/p): 016-9712549, Tel (Office): 609-6688533

Fax: 609-6687896

Email:wanrohani@unisza.edu.my ethnic group to develop gout in Malaysia was Malay with $72 \%$, followed by Indian (20\%) and Chinese $(8 \%)$ likelihood. ${ }^{8}$

It is believed that interaction between genetic and environmental factors play a pivotal role in gout development. The evidence from genome wide association studies showed several genes yielding a significant $p$ value in gouty cohort compared to control cohort. These targeted genes include SLC2A9, $A B C G 2, S L C 22 A 12$ and URATE1 genes where they are particularly expressed at the proximal tubule of kidney and are involved in excretion and reabsorption of uric acid. Polymorphisms of these genes have been observed to associate with gouty arthritis susceptibility among Caucasians and Asian population.

Recently, a series of studies $9,10,11$ have found significant correlations between mutations in the SLC2A9 gene and alterations in plasma urate concentration. Solute carrier family 2, member 9 (SLC2A9), also known as glucose transporter 9 (GLUT 9) is a uric acid transporter at the renal proximal tubule. This transporter involves in reabsorption and excretion of uric acid, thus influencing serum urate levels. Even though correlation between most of these mutations appear to have small significance, the elevation of plasma urate was observed approximately to be $10 \%$. Several SLC2A9 polymorphisms have shown association with gout, providing approximately $3 \%$ variance of this gene in affecting serum urate level.

To support the findings, genome-wide studies have also revealed association of SLC2A9/GLUT9 gene variants with gout in various populations such as Caucasian population, ${ }^{10}$ Japanese men, ${ }^{12} \mathrm{New}$ Zealand's 
Maori and Pacific Islanders, ${ }^{13}$ Han Chinese and Solomon Islanders. ${ }^{14}$ Several recent genome-wide association studies (GWAS) and follow-up studies identified that genetic variants of SLC2A9 had a role in affecting urate acid concentration. $9,10,11,15,16,17,18,19$ and susceptibility to gout. ${ }^{12,14,15,17,20,21}$ Dehghan and colleagues confirmed the association between polymorphism in SLC2A9 with both serum uric acid level and risk of gout in recent study of three cohorts; in the Framingham cohort and in two of the Rotterham cohorts. ${ }^{11}$

\section{MATERIALS AND METHODS}

\section{Objective}

This study was conducted in order to associate SLC2A9 variants with gout among Malay males in Malaysia.

\section{Subject recruitment}

Seventy eight gouty patients who visited the Medical Specialist Clinic at Hospital Universiti Sains Malaysia (HUSM) and eighty two normal subjects were recruited and given consent for the study. All the patients met the American College of Rheumatology (ACR) criteria 1977 and diagnosed with gout. Cases were all Malay descendants; aged 17-71 years old and who satisfied the criteria more than 6 out of 13 of ACR diagnosis criteria. Likewise, 100 Malay subjects with no selfreported history of gout or other serious illnesses were recruited. All patients and controls were Malays, selected amongst the Malaysian population. Phenotypic characteristics included demographic data and clinical parameters (tophi and disease-related complications). Clinical measurement for serum urate and creatinine were taken from serum specimens of gouty patients and analysed by standard procedures in the Chemical Pathology Laboratory, HUSM.

\section{SNP Genotyping}

Genomic DNA was extracted from $3 \mathrm{ml}$ of peripheral blood. Three SNPS of SLC2A9 were genotyped that were assigned as rs3733591, rs5028843 and rs11942223 using polymerase chain reaction-restriction fragment length polymorphism (PCR-RFLP) technique. rs3733591 using primers ATGGTGACAATCACGGTGAC and TCCAAACGTTCTTGGGTAAAG that result in cleavage of the 153 bp product into $90 / 63$ bp fragments using BSH 1236; rs5028843 using primers AAGTGATTGTGTTGATGGCC and AGGGCTTGACCTTAAAATCT that result in cleavage of the 319 bp product into 220/99 bp fragments using Rsa1; and rs11942223 using primers AGCCTCAGAGAAGTGAAGTAAC and AAATTGCTCTGACTTGGGAGTTAGGAAGTC that result in cleavage of the $101 \mathrm{bp}$ product into 71/30 bp fragments using aTaq 1 . The PCR amplicons were run on $2 \%$ Agarose gel electrophoresis and visualized under UV transilluminator. One digested product from each genotype of SNPs was sequenced for validation.

\section{Statistical analysis}

The priori sample size calculation was based on previous gout data on New Zealand Polynesians, 13,22 Chinese and Solomon Islander population ${ }^{14}$ and Japanese population ${ }^{12}$ which gave overall minor allele frequency (MAF) of 0.425 with OR of 1.146 for rs 3733591 projected $10 \%$ of power with 78 cases. Overall MAF of 0.929 with OR of 1.802 for $r s 11942223$ projected $28 \%$ of power with 78 cases. However average MAF of 0.124 with OR of 0.13 for rs 5028843 was obtained from Hollis-Moffatt et al $2009^{21}$ with smaller cases size $(n=125)$ among Maoris and Pacific Islanders generated $99 \%$ of power with 78 Malay male gouty patients. Hardy-Weinberg equilibrium was calculated using SHEsis online software (http:// analysis.bio-x.cn/myAnalysis.php) for cases and control providing odd ratios (ORs) and 95\% confidence interval $(95 \% \mathrm{Cl})$ with $\mathrm{P}$ value of $<0.05$ is considered statistically significant for single association. Publicly online software, SHEsis was utilized to measure the association of SLC2A9 variants with gout development for cases and controls providing odd ratios (ORs) and $95 \%$ confidence interval $(95 \% \mathrm{Cl})$ with $\mathrm{p}$ value of $<0.05$ is considered statistically significant.

\section{RESULTS}

Single association analysis of $r s 3733591$ and $r s 5028843$ revealed significant association with $p$ value of 0.020 and 0.036 , respectively while $r s 11942223$ did not yield a significant value of association with $p=0.080$. The minor allele of these three variants showed a trend towards susceptibility based on odd ratios more than $1(\mathrm{OR}=3.547,3.667$ and 2.732, respectively) (Table I). 
Table I: Association analysis of three SNPs of SLC2A9 with gout in Malay males

\begin{tabular}{|c|c|c|c|c|c|c|c|c|}
\hline \multirow{2}{*}{$\begin{array}{l}\text { SNPs } \\
r s 3733591\end{array}$} & & \multicolumn{3}{|c|}{ Genotype frequency } & \multirow{2}{*}{\begin{tabular}{|l}
${ }^{*} \mathrm{MAF}$ \\
$\mathrm{C}$ \\
\end{tabular}} & \multirow{2}{*}{$\begin{array}{c}{ }^{*} \mathrm{OR} \\
{[95 \% \mathrm{Cl}]}\end{array}$} & \multirow{2}{*}{\begin{tabular}{|l}
$\begin{array}{l}\text { Allelic } \mathrm{p}- \\
\text { value }\end{array}$ \\
\end{tabular}} & \multirow[t]{2}{*}{ HWE } \\
\hline & & TT & CT & CC & & & & \\
\hline & Case & $69(0.841)$ & $12(0.146)$ & $1(0.012)$ & 0.085 & \begin{tabular}{|l}
3.547 \\
{$[1.141-$} \\
$11.022]$
\end{tabular} & 0.020 & 0.569 \\
\hline & Control & $74(0.949)$ & $4(0.051)$ & $0(0.000)$ & 0.026 & & & 0.816 \\
\hline \multirow[t]{3}{*}{ rs5028843 } & & GG & GA & $\mathrm{AA}$ & $A$ & & & \\
\hline & Case & $71(0.866)$ & $11(0.134)$ & $0(0.000)$ & 0.067 & $\begin{array}{c}3.667 \\
{[1.003-} \\
13.402]\end{array}$ & 0.036 & 0.515 \\
\hline & Control & $75(0.962)$ & $3(0.038)$ & $0(0.000)$ & 0.019 & & & 0.863 \\
\hline \multirow[t]{3}{*}{ rs11942223 } & & TT & CT & CC & C & & & \\
\hline & Case & $71(0.866)$ & $11(0.134)$ & $0(0.000)$ & 0.067 & $\begin{array}{c}2.732 \\
\\
{[0.851-} \\
8.769]\end{array}$ & 0.080 & 0.515 \\
\hline & Control & $74(0.949)$ & $4(0.051)$ & $0(0.000)$ & 0.026 & & & 0.816 \\
\hline
\end{tabular}

Association analysis was generated using SHEsis online software.

On further investigation, haplotype $2 / 1 / 2,2 / 1 / 1$, $1 / 2 / 1,1 / 1 / 2$ representing $r s 3733591$, rs5028843 and $r s 11942223$ revealed no association with a trend towards susceptible effect projected by $\mathrm{p}=0.256$ $(\mathrm{OR}=2.536[0.482-13.326]), 0.124 \quad(\mathrm{OR}=3.258[0.666-$
$15.941]), 0.100(\mathrm{OR}=2.914[0.770-11.022])$ and 0.240 $(\mathrm{OR}=2.576$ [0.503-13.189]), respectively. However there was a suggestive evidence of protective effect generated from haplotype $1 / 1 / 1$ with $p$ value of 0.004 (OR=0.324 [0.147-0.716]) (Table II).

Table II: Haplotype analysis of three markers of SLC2A9 with gout in Malay males

\begin{tabular}{|l|l|l|l|l|l|}
\hline haplotype & Case (freq) & Control (freq) & Chi2 & $\begin{array}{l}\text { Pearson's P } \\
\text { value }\end{array}$ & \multicolumn{1}{|c|}{$\begin{array}{c}\text { Odds ratio } \\
{[95 \% \mathrm{Cl}]}\end{array}$} \\
\hline $2 / 1 / 2$ & $5.09(0.031)$ & $1.97(0.013)$ & 1.293 & 0.256 & $\begin{array}{l}2.536[0.482- \\
13.326]\end{array}$ \\
\hline $2 / 1 / 1$ & $6.66(0.041)$ & $2.03(0.013)$ & 2.367 & 0.124 & $\begin{array}{l}3.258[0.666- \\
15.941]\end{array}$ \\
\hline $1 / 2 / 1$ & $8.74(0.053)$ & $3.00(0.019)$ & 1.382 & 0.100 & $\begin{array}{l}2.914 \quad[0.770- \\
11.022]\end{array}$ \\
\hline $1 / 1 / 2$ & & & & & $\begin{array}{l}2.576[0.503- \\
13.189]\end{array}$ \\
\hline $1 / 1 / 1$ & $136(0.829)$ & $147(0.942)$ & 8.397 & 0.004 & $\left.\begin{array}{l}0.324 \\
0.716]\end{array}\right]$ \\
\hline
\end{tabular}

$\mathrm{Cl}$, confidence interval; Sequential row in haplotypes represents Rs3733591, rs5028842 and rs11942223. 


\section{DISCUSSION}

This study is the first attempt to evaluate the potential association between gout and SLC2A9 polymorphisms and haplotypes in Malay male dataset in Malaysia.

Gout is the most common form of inflammatory arthritis that arise from the formation and deposition of multiple needles like uric acid crystals in and around the articular joints. Gouty arthritis has shown to affect mainly men rather than women in many populations in several studies. ${ }^{23}$ The uricosuric agent from estrogen of women is believed to protect women from having gout. Once the women reach the menopausal stage with a decline in estrogen level, they are no longer protected by this uricosuric agent and therefore postmenopausal women too are predisposed to gout development. ${ }^{24}$

SLC2A9 has been recognized as urate transporter at renal proximal tubule that plays a pivotal role in uric acid excretion and reabsorption. Mutation to the transporter may lead to defect in homeostasis, thus influencing serum uric acid level. Several variants of SLC2A9 have showed association with gout in various populations such as in Caucasians, Japanese, Chinese, Pacific Islanders, Maori New Zealand and others. 10,12,13,14,21,22 The association data from this study was shown consistent with the previous studies in different population where rs3733591 and rs5028843 revealed a significant association with gout among Malay males in Malaysia. A fair association was also noted for rs 11942223 with a trend towards susceptible effect.

The C-allele of the nonsynonymous rs3733591(R265H) variant of SLC2A9 has conferred risk for gout in other Asian populations such as Hans Chinese, Solomon Island and Japanese cohorts that is consistent with our Malay dataset. ${ }^{12,14}$ However there was no evidence of association observed in New Zealand Caucasian and Polynesian as C-allele of rs3733591 might give a weaker effect on gout in these population compared to the Asian and Melanesian populations. ${ }^{13}$ rs5028843 was also noted to have association with gout in Maori and New Zealand Caucasian with minor allele conferred protective effect that is contrary to our data (more pronounced as susceptibility effect), ${ }^{21}$ This might be explained by the genetic drift that contributes to genetic heterogeneity in population stratification. Risk T-allele of rs11942223 also conferred susceptibility to gout in New Zealand Caucasian and New Zealand Polynesian ${ }^{22}$ which is consistent with our study even though a fair association was observed in Malay male dataset.

Captivated by this promising data, three-marker haplotype association analysis was performed in order to determine the role of haplotypes of SLC2A9. The association data showed major allele of the variants (haplotype 1/1/1) can protect individuals from having gout. Even though other haplotypes $(2 / 1 / 2$, $2 / 1 / 1,1 / 2 / 1,1 / 1 / 2$ ) did not show association, the odds ratio described the susceptible effect from these haplotypes. However, replication study should be performed in a larger sample size to project a larger power of study and to ascertain a validation data with functional analyses with a well-designed clinical investigations to shed light on potential biological mechanism that links between the SLC2A9 polymorphisms with the risk of gout and thus may provide a selective biomarker development for a better treatment of gout therapy regime.

\section{CONFLICT OF INTEREST}

The authors declare that they have no competing interests.

\section{ACKNOWLEDGEMENTS}

This work was supported by the Universiti Sains Malaysia Short Term Grant (No grant: 304/ PPSP/61311050) and the study protocol conforms to the ethical guidelines of the Research Review Board and Human Research Ethics Committee of Universiti Sains Malaysia, Kelantan, Malaysia [USMKK/PPP/JEPeM [234.3.(01)]]

\section{REFERENCES}

1. Wortmann RL. Gout and hyperuricemia. Current Opinion Rheumatology 2002;14:281-6.

2. Choi HK, Mount DB, Reginato AM. Pathogenesis of gout. Ann Intern Med 2005; 143:499-516.

3. Arromdee E, Michet CJ, Crowson CS, O'Fallon WM, Gabriel SE. Epidemiology of gout: is the incidence rising? J Rheumatology 2002; 29:24036.

4. Klemp P, Stansfield SA, Castle B \& Robertson MC (1997). Gout is on the increase in New Zealand. Ann Rheum Dis 1997; 56:22-6.

5. Dalbeth N, Collis J, Gregory K, et al. Tophaceous joint disease strongly predicts hand function in patients with gout. Rheumatology 2007; 46:18047.

6. Wallace KL, Riedel AA, Joseph-Ridge N, Wortmann R. Increasing prevalence of gout and hyperuricemia over 10 years among older adults in a managed care population. J Rheumatology 2004; 31:1582-7.

7. Chang HY, Pan WH, Yeh WT, Tsai KS.

Hyperuricemia and gout in Taiwan: results from the Nutritional and Health Survey in Taiwan (1993-1996). J Rheumatology 2001; 28:1640-6.

8. Mohd A, Das Gupta E, Loh YL, et al. Clinical characteristics of gout: A hospital case series. Malaysian Family Physician 2011; 6:2-3.

9. Wallace C, Newhouse SJ, Braund P, et al. Genome-wide association study identifies genes for biomarkers of cardiovascular disease: serum urate and dyslipidemia. Am J Hum Genet 2008; 82:139-49.

10. Li S, Sanna S, Maschio A, et al. The GLUT9 gene is associated with serum uric acid levels in Sardinia and Chianti cohorts. PLoS Genet 
2007; 3:e194.

11. Dehghan A, Kottgen A, Yang Q, et al. Association of three genetic loci with uric acid concentration and risk of gout: a genome-wide association study. Lancet 2008; 372:1953-61.

12. Urano W, Taniguchi A, Anzai N, et al. Association between GLUT9 and gout in Japanese men. Ann Rheum 2010, 69:932-3.

13. Hollis-Moffatt JE, Gow PJ, Harrison AA, et al. The SLC2A9 nonsynonymopus Arg256His variant and gout: evidence for a population-specific effect on severity. Arthritis Research \& Therapy 2011, 13:R85.

14. Tu HP, Chen CJ, Tovosia S, et al. Associations of nonsynonymous variant in SLC2A9 with gouty arthritis and uric acid levels in Han Chinese subjects and Solomon Islanders. Ann Rheum 2009; 69:887- 90.

15. Vitart V, Rudan I, Hayward C, et al. SLC2A9 is a newly identified urate transporter influencing serum urate concentration, urate excretion and gout. Nat Genet 2008; 40:437- 42.

16. Doring A, Gieger C, Mehta D, et al. SLC2A9 influences uric acid concentrations with pronounced sex-specific effects. Nat Genet 2008; 40:430-6.

17. McArdle PF, Parsa A, Chang YP, et al. Association of a common nonsynonymous variant in GLUT9 with serum uric acid levels in Old Order Amish. Arthritis Rheum 2008; 58:2874-81.

18. Brandstatter A, Kiechl S, Kollerits B, et al. Sexspecific association of the putative fructose transporter SLC2A9 variants with uric acid levels is modified by BMI. Diabetes Care 2008; 31:1662-7.

19. Zemunik T, Bohan M, Lauc G, et al. Genomewide association study of biochemical traits in Korcula Island, Croatia. Croat Med J 2009; 50:23-33.

20. Stark K, Reinhard W, Neureuther K,et al. Association of common polymorphisms in GLUT9 gene with gout but not with coronary artery disease in a large case-control study. PLoS One 2008; 9:e1948.

21. Hollis-Moffatt JE, Xu X, Dalbeth N, et al. Role of the urate transporter SLC2A9 gene in susceptibility to gout in New Zealand Maori, Pacific Island and Caucasian case-contril sample sets. Arthritis \& Rheumatology 2009; 60:348592.

22. Phipps-Green AJ, Merriman ME, Topless R, et al. Twenty-eight loci that influence serum urate levels: analysis of association with gout. Clinical and Epidemiological Research 2014; 1:1-7.

23. Saag KG, Choi HK. Epidemiology, risk factors, and lifestyle modifications in gout. Arthritis Res Ther 2006; 8:S2.
24. Yahyaoui R, Esteva I, Haro-Mora JJ, et al. Effect of long term administration of cross-sex hormone therapy on serum and urinary uric acid in transsexual persons. $\mathrm{J}$ Clin Endocrinol 
HE INTERNATIONAL MEDICAL JOURNAL MALAYSIA 